\title{
Mechanism of Ostwald Ripening in 2D Physisorbed Assemblies at Molecular Time and Length Scale by Molecular Dynamics Simulations
}

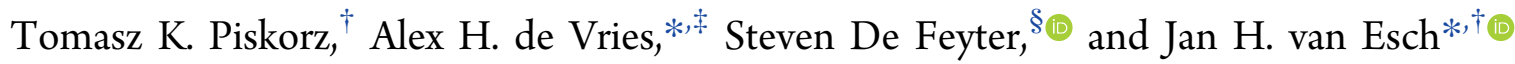 \\ ${ }^{\dagger}$ Advanced Soft Matter, Chemical Engineering, Delft University of Technology, van der Maasweg 9, 2629 HZ Delft, The \\ Netherlands \\ ${ }^{\ddagger}$ Groningen Biomolecular Sciences and Biotechnology Institute and Zernike Institute for Advanced Materials, University of \\ Groningen, Nijenborgh 7, 9747 AG Groningen, The Netherlands \\ ${ }^{\S}$ Department of Chemistry, KU Leuven, Celestijnenlaan 200F, 3001 Leuven, Belgium
}

\section{Supporting Information}

\begin{abstract}
Ostwald ripening can improve the long-range order of self-assembled monolayers by the growth of large domains and disassembly of smaller ones. Here, coarse-grained molecular dynamics simulations are used to study the dynamics of the stable assembly and the coarsening of defects of physisorbed monolayers of long-chain functionalized alkanes. Our results show that the partial desorption from the surface of one or more adsorbent molecules is the essential process that allows other adsorbent molecules to rearrange on the surface and thereby improve alignment. We also show that the ripening process is faster at higher temperature because the rate of partial desorption is higher.
\end{abstract}

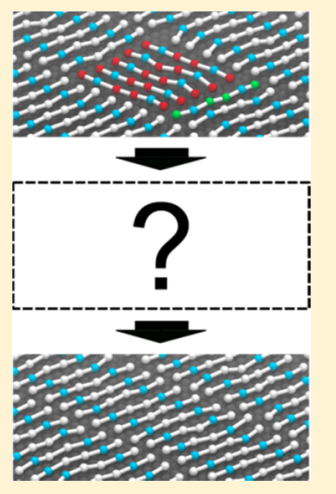

\section{INTRODUCTION}

Long-range ordered physisorbed self-assembled monolayers on a substrate surface are promising for building controlled nanopatterned structures as an alternative to conventional topdown methods currently used in industry. ${ }^{1-4}$ However, obtaining perfectly aligned structures is still a challenging task. ${ }^{5}$ The alignment of the final structure can be improved by Ostwald ripening, in which large domains grow at the expense of small ones. ${ }^{6-8}$ A better understanding of the process could improve control over the alignment of the assemblies.

The Ostwald ripening of physisorbed self-assembled monolayers has been studied experimentally using scanning tunneling microscopy (STM) by Stabel et al. ${ }^{6}$ They have shown that the process occurs while the adsorbents remain on the surface and is reaction-controlled, i.e., the limiting step is desorption from one domain and adsorption to another. Such a reordering on the surface was directly observed using STM. ${ }^{9}$ This explanation assumes that a liquidlike zone exists between domains in which a molecule can rearrange and adsorb to a domain. However, more stable domains with tightly packed interfaces ${ }^{7}$ also can undergo coarsening. For example, for an alkylated porphyrin on graphite, the growth of one domain at the expense of another was shown to occur only at a defect. ${ }^{9}$ Note that the term "coarsening" in the context of domain formation and growth refers to the appearance of large, long-range ordered domains. Experimental studies of the molecular details of the coarsening process by STM are limited by its spatial and temporal resolution, as well as by potentially strong interactions between the probe and the adsorbent. $^{10}$

In this work, we provide a molecular-level description of the coarsening process. Since the process happens on a short time scale, we choose to use molecular dynamics simulations to study these systems, which allow high resolution in time and space.

\section{RESULTS AND DISCUSSION}

Self-Assembly on Graphite. We utilize a recently developed variant of the Martini model to simulate adsorption from a solution and subsequent self-assembly of molecules on a graphite surface at near-atomistic resolution. ${ }^{11}$ Molecules of a six-bead adsorbent, representing a long-chain alkane functionalized with amide groups (Figure 1a), were randomly placed in a simulation box containing a graphite flake (Figure $1 \mathrm{~b}$ ), and solvent, phenyloctane, was added. In repeated simulation runs at $298 \mathrm{~K}$, the adsorbent preferentially adsorbs on the surface and forms an ordered structure after about $2 \mu \mathrm{s}$, usually consisting of several large domains (Figure 1c). The mechanism of this process has been described elsewhere. ${ }^{12}$ Simulation of the system for another $5 \mu$ s leads to the graphite surface being covered by a single domain (Figure 1d), which has some

Received: July 5, 2018

Revised: September 18, 2018

Published: September 25, 2018 

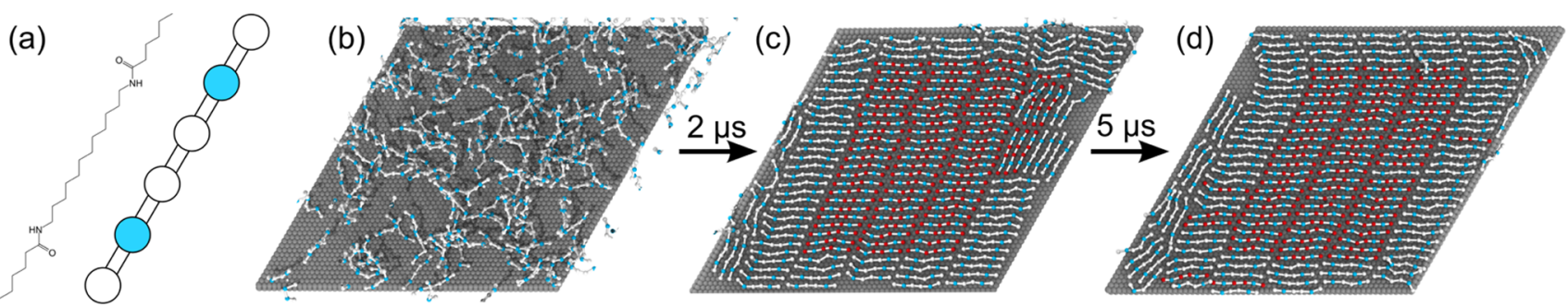

Figure 1. (a) Molecule studied in this work and its coarse-grained representation. (b) Snapshot of the initial system: graphite flake and randomly distributed adsorbent in 1-phenyloctane (not shown for clarity), (c) snapshot of an initial self-assembled monolayer after $2 \mu$ s of simulation, (d) snapshot of a well-ordered self-assembled monolayer after additional $5 \mu \mathrm{s}$ of simulation. Inner lamellae (indicated by red; $2 \mathrm{~nm}$ from the edge of the graphite flake) do not undergo substantial structural changes. In contrast, molecules on the edge of graphite flake stay dynamic.

(a)
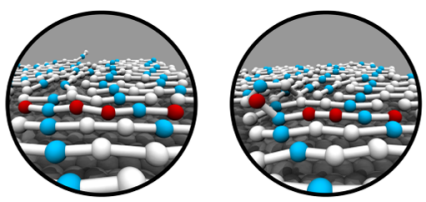
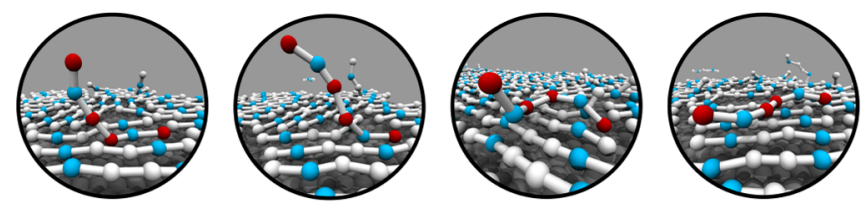

(b)

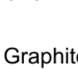

layer

(c)

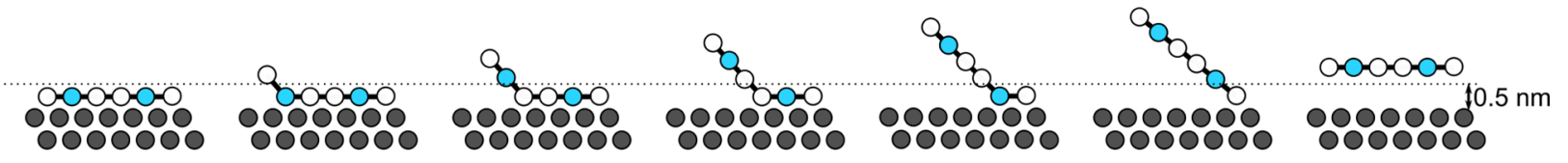

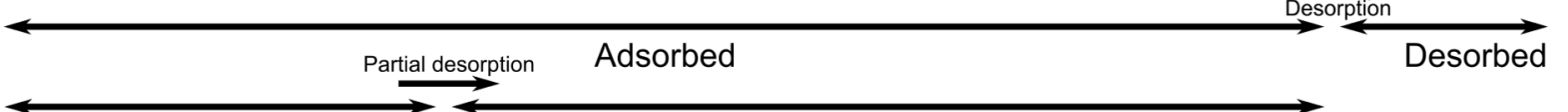

Fully adsorbed

(d)

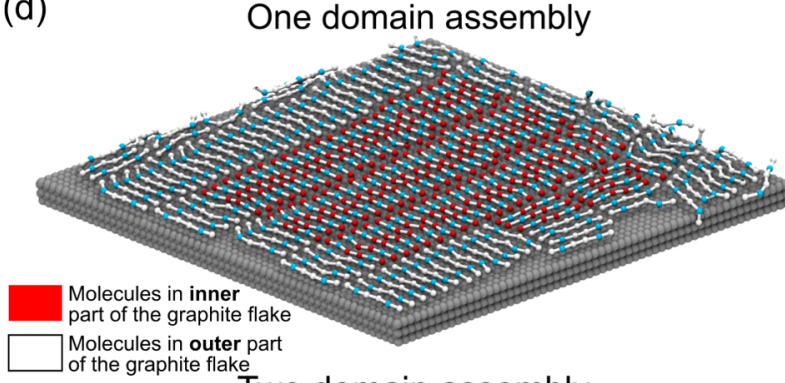

(e)

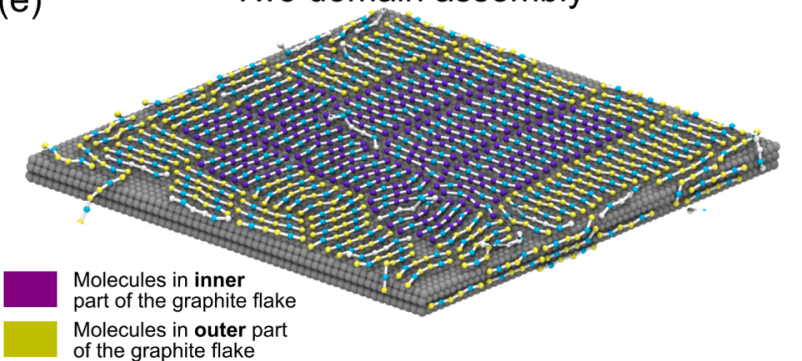

Partially adsorbed

(f)

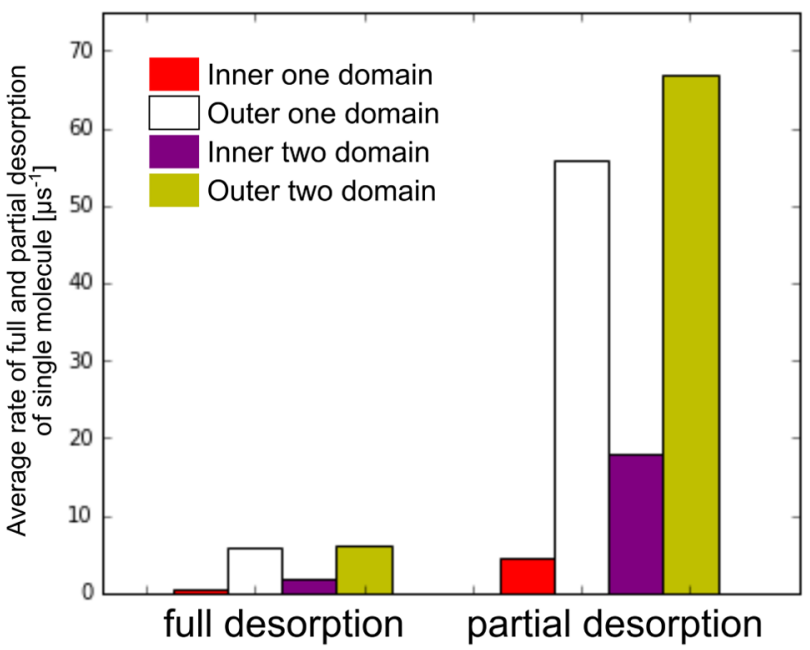

Figure 2. (a) Snapshots and (b) schematic representation of examples of partially desorbed molecules. (c) Classification of different states and processes analyzed in this work. Snapshots of initial (d) one-domain and (e) two-domain structures of assemblies on graphite. For analysis, two parts of the assembly were distinguished: inner (molecules more than $2 \mathrm{~nm}$ away from the graphite edge, indicated by red for one-domain assembly and purple for two-domain assembly) and outer (molecules within $2 \mathrm{~nm}$ from the graphite edge, indicated by white for one-domain assembly and yellow for twodomain assembly). (f) Rates of full and partial desorption of molecules from a self-assembled monolayer on the graphite flake during $5 \mu$ s of simulations.

remaining packing defects and is surrounded by a small number of molecules near the edge of the flake that have a different orientation, likely due to a mismatch between the width of the lamellae and the size of the flake.

Dynamics of the Self-Assembled Monolayer. To better understand the dynamics of the defects, we first analyzed the dynamics of the molecules in a single, stable, and well-ordered domain, as illustrated in Figure $2 \mathrm{~d}$. We measured the rates of full and partial vertical desorption from and readsorption to the surface during a period of $5 \mu \mathrm{s}$. As an example, Figure 1c,d illustrates the initial and final structures of such a run. In partial vertical desorption, two or more beads lift off the surface, but at 
least one bead remains on the surface (see Methods). To give a better impression of the types of desorption molecules display, Figure 2a shows some examples of partially desorbed molecules pointed out in snapshots taken from the simulations (and for clarity Figure $2 \mathrm{~b}$ shows its schematic representation). It can be seen that one or more beads from a molecule can be lifted from the surface at different positions within the chain. The classification of different states and processes is schematically represented in Figure 2c. Figure 2f summarizes the results (additional results and study of different types of molecules can be found in Figure S1 and Table S1). The rates are represented by frequencies, that is, how often (on average) an adsorbed molecule vertically fully desorbs or partially desorbs. The process of full vertical desorption is observed rarely and mostly at the edges of the graphite flake. The inner part of the assembly is especially stable (compare Figure 1c,d). The full desorption of these molecules is $\sim 10$ times less frequent than desorption of molecules close to the edge of the graphite flake.

Although it appears that the inner part of the assembly is static, a closer look reveals that partial desorption is frequent (the rate is $\sim 10-20$ times larger than that of full desorption). The lifetime of this partially desorbed unstable state is short (in all simulations $\sim 1$ ns; see Table S1), and molecules tend to readsorb quickly on the surface.

It is noteworthy that the dynamics of the assemblies depends on temperature and the chemical nature of the adsorbents. By increasing the temperature, the monolayer becomes more dynamic, which can be observed as the increased rate of full and partial desorptions (see Table S1).

The dynamics of the molecules in the single domain was contrasted with that in assemblies composed of two domains separated by a domain boundary (Figure 2e), which shows that the partial desorption in the latter case is more frequent (see Figure 2f). This finding can explain "fuzziness" of domain boundaries observed on STM images. ${ }^{8}$ At the domain interface, molecules have different orientations, which hinders stabilization by alignment of the functional groups, resulting in more frequent partial desorption. An increased rate of desorption/ readsorption of molecules near defects was also observed experimentally for systems of long-chain functionalized alkanes. $^{13}$

Ostwald Ripening. Ostwald ripening (in the context of physisorbed self-assembled monolayers sometimes referred to as self-healing or domain coarsening) is a process in which large domains grow at the expense of small ones. ${ }^{6}$ The thermodynamic force driving this process is the decrease of interfacial energy generated by different domain boundaries. In our earlier work, most of the independent repeated self-assembly simulations led to well-ordered lamellae in an equilibrated state, but we observed several types of defects, with long lifetimes. Here, we elaborate on the domain coarsening of two types of defects: (a) two large domains that both occupy a large area of graphite and (b) a small defect surrounded by a large domain.

First, we analyzed coarsening of two domains with different orientations, which span on the entire graphite flake. Figure $3 \mathrm{a}$ shows a zoomed-in image on the domain boundary, and the full graphite surface can be found in Figure S5. Molecules are colored on the basis of their initial membership of one of the two domains. As mentioned before, the structure is most dynamic on the interface of two domains. We observed that in both temperature regimes one of the domain orientations starts to dominate. Figure $3 b, c$ shows the changes in organization at
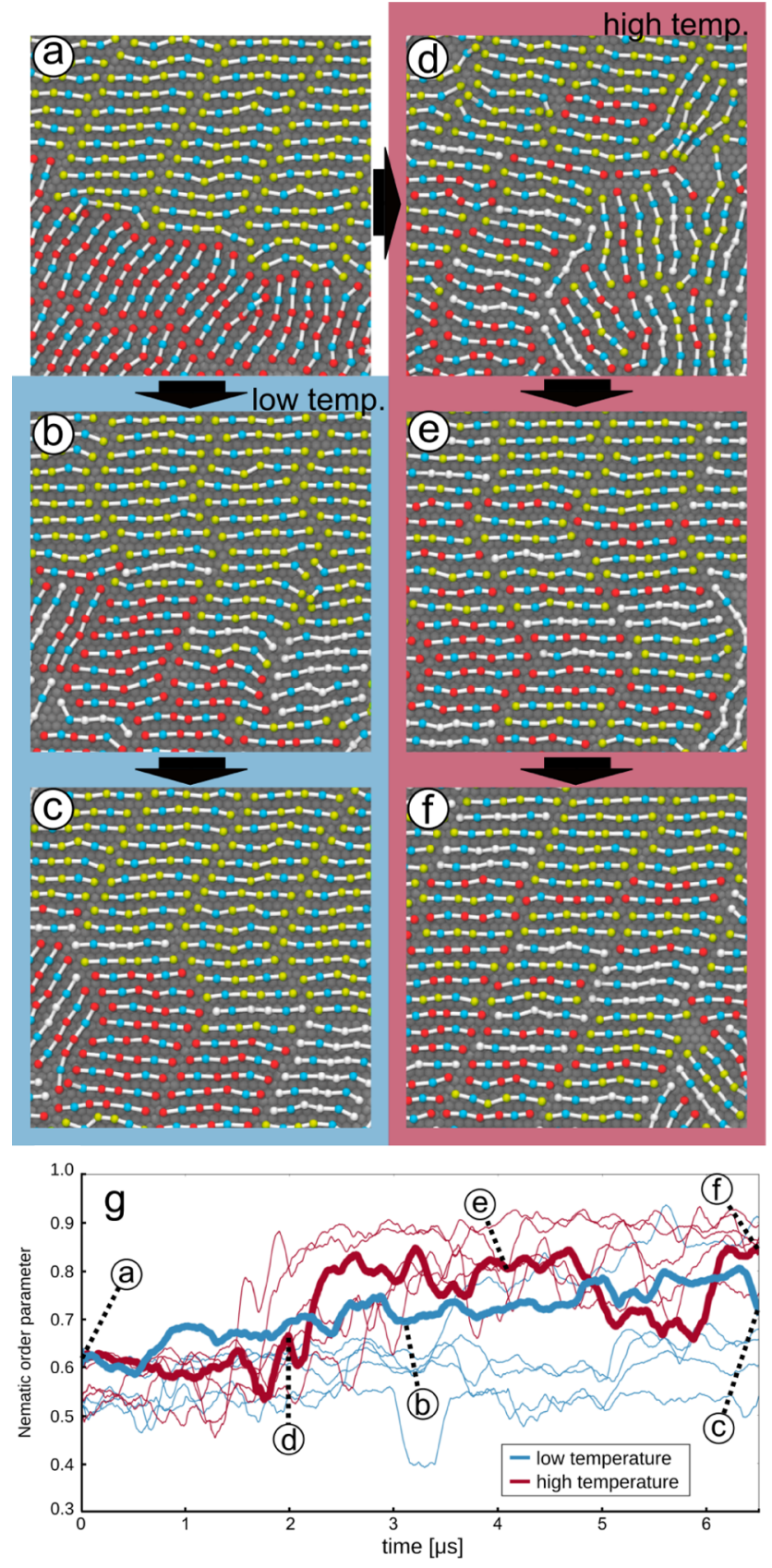

Figure 3. Reorganization of two domains with a different orientation. (a-f) Snapshots of two representative trajectories. The color of the molecules (red and yellow) indicates initial membership to one of the original domains, whereas white color indicates molecules that are not a part of the original domains (they originate in the supernatant or from the edges of the flake). At both temperatures, the domains rearrange, but at lower temperature (b, c), they do not mix extensively, whereas at higher temperature $(\mathrm{d}-\mathrm{f})$, they do. (g) Nematic order parameter for the process at room temperature (blue) and high-temperature regime (red) for six independent simulations. The bold lines represent the nematic order parameter for the simulations from which the snapshots were taken. At higher temperature, molecules align faster.

lower temperature and Figure $3 \mathrm{~d}-\mathrm{f}$ at higher temperature for one of six independent simulations of this process. At low temperature, the coarsening process occurs in several distinct stages. Local fluctuation causes one of the lamellae of one domain to grow at the expense of the second domain. One by one, lamellae start to extend by rearrangement of the second 
domain, until the entire surface is covered by a single domain. The increased alignment can be seen as small jumps in the nematic order parameter plot (see Figure $3 g$, blue line; see Methods). In the higher-temperature regime, this transformation is more dramatic. The instability on the domain boundary progresses through the major part of one of the domains, resulting in disassembly of the domain into partially ordered clusters. These clusters restructure and align with the second domain, covering the entire flake. This can be seen in the nematic order parameter as a small decrease (disassembly of one of the domains) followed by a sudden increase that leads to the ordered structure (see Figure $3 \mathrm{~g}$, red line). At both temperatures, kinks occur in the lamellae structure, forming "zig-zag" patterns. Such structures have been observed among others in docosylether physisorbed on graphite and were characterized as the effect of a pair of twin boundaries between lamellae. In the study by Padowitz et al., ${ }^{13}$ motion of the pair of twin boundaries was observed. A twin boundary is the result of the change in displacement or shift between two neighboring molecules within a lamella, in which the angle between the lamellar axis and the molecular axis is not $90^{\circ}$, but $60^{\circ}$. In our model, there is such a displacement between neighboring molecules because of the coarse-grained nature of the beads. The lateral interaction between neighbors is more favorable if the neighboring molecules are displaced by half a bond length because a bead interacts with two neighboring beads instead of one. More discussion can be found in Supporting Information (SI).

Although both assemblies show an increased order, the composition of the final structure is different. At the lower temperature, the reorganization is local and molecules rearrange on the surface, i.e., without full desorption. In the final structure, it can be clearly seen that the assembly was formed from different domains by reorientation of the molecules in one of the domains. In contrast, the rearrangement in the highertemperature regime leads to extensive mixing of the molecules in the final structure. This indicates that significant rearrangement of the molecules was involved. By looking at the trajectories, several types of molecular rearrangements can be observed. It is difficult to quantitatively classify these events; we feel that the nematic order parameter is a useful measure to monitor the increased ordering. Movies of several rearrangement events are provided in SI.

The second type of commonly observed defect is one in which in the middle of the well-ordered structure a small defect is incorporated, as shown in Figure 4a (snapshots of the whole system can be found in SI). We constructed a single large domain with one such defect in order to be able to study only the rearrangement of the defect. This way, we avoided all of the issues present in the two-domain study, such as edge effect, imperfect packing, etc. Defects that are close to an edge of the graphite flake rapidly disappear due to increased mobility on the edge of the flake; see SI. We ran several instances of simulations and observed different behavior at different temperatures (Figure $4 \mathrm{~b}-\mathrm{i}$ ). Similar as for coarsening of two domains, the process is slower at the lower temperature (Figure $4 \mathrm{k}$ ). At the lower temperature, reorganization occurs on the surface of the graphite flake; molecules partially vertically desorb, resulting in free space on the graphite flake for other molecules to laterally desorb from one domain and readsorb on the other. At the higher temperature, the partial vertical desorption occurs more often. This results in higher lateral mobility of the molecules on the surface, effectively speeding up the healing. It is worth mentioning that vacancies on the graphite flake were not
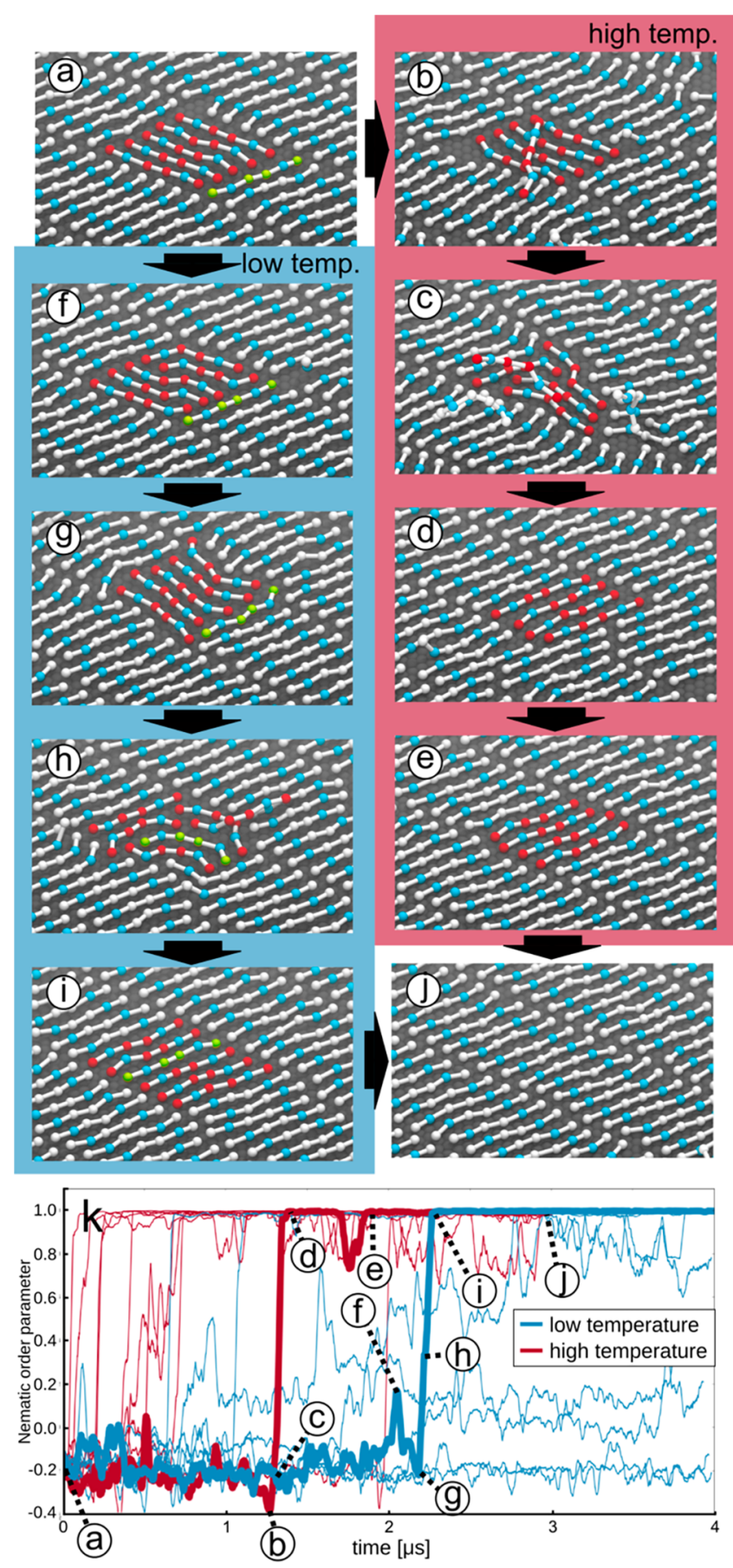

Figure 4. Reorganization of a small defect surrounded by a large domain. Selected beads of the molecules that constitute the defect are shown in red. (a) Snapshot of starting small structure defect. (b-e) Representative snapshots of Ostwald ripening at higher temperature and $(\mathrm{f}-\mathrm{i})$ at lower temperature (the molecule indicated by yellow takes part in the final healed structure, although at the beginning, it was part of the well-ordered lamella), $(j)$ representative snapshot of the healed structure. (k) Nematic order parameter of the defect molecules with respect to the large-domain director for the process at lower temperature (blue) and higher temperature (red). The bold lines represent the trajectories from which snapshots were taken; the snapshots are indicated by the letters. At high temperature, the process starts early and it is fast, whereas at room temperature, it takes longer to begin the process and when initiated it takes a longer time to complete. 
observed; the area freed by (partially) desorbing molecules is occupied by beads of adsorbent or solvent molecules within the time between snapshots (which is $30 \mathrm{ps}$ ).

Finally, simulations of an asymmetrically functionalized molecule (see SI) showed that the rearrangement process of the isolated domain occurs in a similar manner as for the symmetric molecule described above. Although partial desorption rates for different molecules differ, we do not have enough statistics to make statements about how these affect the rate of the rearrangement of the isolated domain.

Mobility. Our simulations at different temperatures suggest that the rate of coarsening depends on the partial vertical desorption of molecules from the surface, which is enhanced near the edge of the graphite flake, at domain boundaries, and at a higher temperature. Partial vertical desorption is expected to increase the lateral mobility of molecules on the surface because packing is less tight. We previously also observed that when the surface is not completely covered, the lateral mobility of adsorbent on the surface is high. ${ }^{12}$ To investigate the influence of the partial desorption on the surface mobility, we imposed forces in the $z$-direction (the normal vector to the graphite plane) restraining the adsorbed molecules to the surface, thereby suppressing (partial and complete) desorption. For a single molecule, the restraining force can be related to adsorption energy. We randomly removed $\sim 10 \%$ molecules from the system and observed changes in the mobility. An increase in the restraining force leads to a drop in mobility (see Table S2 for quantification). Thus, the lateral mobility of the molecules on the surface strongly depends on the level of partial desorption. Our simulations are consistent with the view that molecules do not need to fully desorb from the surface to enable coarsening; the coarsening process is, however, slowed down considerably if the molecules are more tightly bound to the surface.

\section{CONCLUSIONS}

In this work, we systematically studied the mechanism and the parameters influencing the Ostwald ripening process of selfassembled monolayers. We observed that molecules are more mobile on the interface between different domains and they tend to partially vertically desorb from the surface. Partial vertical desorption allows Ostwald ripening to take place: molecules can laterally desorb from one domain, laterally diffuse, and adsorb on another. Since the rate of partial desorption is higher on the interface, in the long term, the defects promote coarsening. In all of the simulations, increasing the temperature resulted in speeding up the healing of structure. This is caused by increased desorption from the substrate. However, a higher temperature also results in a substantial mixing of the adsorbed molecules on the surface. If the assembly consists of identical molecules, as in this work, this effect is irrelevant; however, it can be crucial for assemblies consisting of various molecules and must be taken into account. Although we did not follow the effect of different solvents here, we expect it to be similar to the temperature effect; a different solvent could influence partial desorption from the substrate, thus speeding up or hindering the healing. We believe that all of the presented results give a clear picture of the mechanism of Ostwald ripening.

\section{METHODS}

Coarse-Grained Model. Simulations were performed using Gromacs 5 software ${ }^{14}$ with the Martini coarse-grained force field. ${ }^{15}$ The Martini force field, initially developed for lipids, was adapted rapidly for other biomolecules ${ }^{15,16}$ and recently to adsorption and self-assembly of small organic molecules on graphite. ${ }^{11}$ As a rule of thumb, the force field represents the system by treating four nonhydrogen atoms as one bead (except for atoms inside a ring, where mapping is $2: 1$ ). Here, we studied a six-bead molecule with two polar beads, which can be viewed as a coarse-grained representation of $N, N^{\prime}$-dodecanomethylenebishexanoamide. Results for other adsorbents can be found in the SI. Self-assembly on a graphite surface of these molecules has been studied in the past. ${ }^{17}$ The molecules were randomly distributed in the system and solvated in phenyloctane. The molecules adsorbed on the surface and formed long-range ordered lamellar structures, which were aligned with the underlying graphite layer (see SI). After $2 \mu \mathrm{s}$ of simulations, an ordered structure formed on a graphite flake. Most often a single, well-ordered domain was formed. However, in some instances, we observed several domains. We used them to study their coarsening by further simulation up to $6.5 \mu \mathrm{s}$. For selfhealing of the small defect, a well aligned long-range ordered structure was used. Five molecules in the middle of the structure were rotated by $60^{\circ}$. Then, the simulations were carried out for another $4 \mu$ s. All simulations were run at two temperatures: 298 $\mathrm{K}$, referred here as lower temperature, and $308 \mathrm{~K}$, referred as higher temperature. We intentionally avoid referring to the absolute temperatures because in general it is difficult to interpret temperature scales in coarse-grained models. ${ }^{18} \mathrm{We}$ also performed simulations at $318 \mathrm{~K}$, at which the long-range ordered structure completely disappears (see SI).

Analysis of Monolayer. Vertical desorption was analyzed as two separate telegraph processes ${ }^{19}$ describing desorption and partial desorption, respectively. For analysis of desorption, molecules were assigned to one of two states: adsorbed (all beads are adsorbed) or desorbed (all beads are desorbed). A single bead is considered adsorbed if it is within $0.5 \mathrm{~nm}$ from the graphite surface. The residence time of an adsorbed state was calculated, and from it, the rate of desorption, i.e., the change from the adsorbed to desorbed state, was calculated. Similar calculations were performed for the partial desorption process, in which molecules were assigned to one of three states: fully adsorbed (five or six beads adsorbed), partially adsorbed (one to four beads adsorbed), and fully desorbed (all beads desorbed). The residence time of a fully adsorbed state was calculated, and from it, the rate of partial desorption, i.e., change from the fully adsorbed state to partial desorbed state, was calculated. Details of this analysis can be found in SI.

Nematic Order Parameter. This parameter quantifies the alignment of a structure. To calculate the parameter, first, the preferred orientation (director) has to be found. This is achieved by finding eigenvectors of the matrix

$$
Q=\left[\frac{1}{2} \sum_{j}\left(3 d_{j, a} d_{j, b}-1\right)\right]_{a, b \in(x, y, z)}
$$

where $d$ denotes the molecular vector (vector connecting the two ends of molecule) of molecule $j$ and the sum runs over all adsorbent molecules. The eigenvector with the largest eigenvalue is called the director, $D$, and it is the preferred orientation of the molecules. The nematic order parameter is calculated as a sum

$$
S=\frac{1}{2 N} \sum_{j}\left(3 \cos ^{2} \alpha_{j}-1\right)
$$


where $\alpha_{j}$ is the angle between the director, $D$, and the molecular vector of $j$ th molecule, $d_{j}$. Low values mean that the molecules are oriented in random directions, and a value close to 1 means that all molecules are aligned in the same direction. In the case of self-healing of small defects, the director was calculated with respect only to the large well-ordered structure.

\section{ASSOCIATED CONTENT}

\section{S Supporting Information}

The Supporting Information is available free of charge on the ACS Publications website at DOI: 10.1021/acs.jpcc.8b06432.

Snapshots of trajectories and results of a study of different functionalized alkanes; details of analysis; Ostwald ripening close to the edge; additional snapshots of trajectories; results for simulations at $318 \mathrm{~K}$ (PDF)

Video of Ostwald ripening of two-domains assembly at $298 \mathrm{~K}$ (MPG)

Video of Ostwald ripening of two-domains assembly at $308 \mathrm{~K}$ (MPG)

Video of Ostwald ripening of small defect at $298 \mathrm{~K}$ (MPG)

Video of Ostwald ripening of small defect at $308 \mathrm{~K}$ (MPG)

\section{AUTHOR INFORMATION}

\section{Corresponding Authors}

*E-mail: a.h.de.vries@rug.nl (A.H.D.V.).

*E-mail: j.h.vanesch@tudelft.nl (J.H.V.E.).

\section{ORCID}

Steven De Feyter: 0000-0002-0909-9292

Jan H. van Esch: 0000-0001-6116-4808

Notes

The authors declare no competing financial interest.

\section{ACKNOWLEDGMENTS}

The authors acknowledge the financial support by the EC 7th Framework Programme Marie Curie Actions via the European Initial Training Network SMARTNET.

\section{REFERENCES}

(1) Hecht, S. Welding, Organizing, and Planting Organic Molecules on Substrate Surfaces - Promising Approaches towards Nanoarchitectonics from the Bottom up. Angew. Chem., Int. Ed. 2003, 42, 24-26.

(2) Joachim, C.; Gimzewski, J. K.; Aviram, A. Electronics Using Hybrid-Molecular and Mono-Molecular Devices. Nature 2000, 408, 541-548.

(3) Kühnle, A. Self-Assembly of Organic Molecules at Metal Surfaces. Curr. Opin. Colloid Interface Sci. 2009, 14, 157-168.

(4) Reed, M. A.; Zhou, C.; Muller, C. J.; Burgin, T. P.; Tour, J. M. Conductance of a Molecular Junction. Science 1997, 278, 252-254.

(5) Metzger, R. M. Unimolecular Electronics and Rectifiers. Synth. Met. 2009, 159, 2277-2281.

(6) Stabel, A.; Heinz, R.; De Schryver, F. C.; Rabe, J. P. Ostwald Ripening of Two-Dimensional Crystals at the Solid-Liquid Interface. J. Phys. Chem. 1995, 99, 505-507.

(7) Samorí, P.; Müllen, K.; Rabe, J. P. Molecular-Scale Tracking of the Self-Healing of Polycrystalline Monolayers at the Solid-Liquid Interface. Adv. Mater. 2004, 16, 1761-1765.

(8) Stabel, A.; Heinz, R.; Rabe, J. P.; Wegner, G.; De Schryver, F. C.; Corens, D.; Dehaen, W.; Sueling, C. STM Investigation of 2D Crystals of Anthrone Derivatives on Graphite: Analysis of Molecular Structure and Dynamics. J. Phys. Chem. 1995, 99, 8690-8697.
(9) Coenen, M. J. J.; Cremers, M.; Den Boer, D.; Van Den Bruele, F. J.; Khoury, T.; Sintic, M.; Crossley, M. J.; Van Enckevort, W. J. P.; Hendriksen, B. L. M.; Elemans, J. A. A. W.; et al. Little Exchange at the Liquid/solid Interface: Defect-Mediated Equilibration of Physisorbed Porphyrin Monolayers. Chem. Commun. 2011, 47, 9666-9668.

(10) Nishitani, N.; Hirose, T.; Matsuda, K. Influence of Multidirectional Interactions on Domain Size and Shape of 2-D Molecular Assemblies. Langmuir 2017, 33, 9151-9159.

(11) Gobbo, C.; Beurroies, I.; De Ridder, D.; Eelkema, R.; Marrink, S. J.; De Feyter, S.; Van Esch, J. H.; De Vries, A. H. MARTINI Model for Physisorption of Organic Molecules on Graphite. J. Phys. Chem. C 2013, $117,15623-15631$.

(12) Piskorz, T. K.; Gobbo, C.; Marrink, S. J.; De Feyter, S.; De Vries, A. H.; Van Esch, J. H.. Manuscript in Preparation.

(13) Padowitz, D. F.; Sada, D. M.; Kemer, E. L.; Dougan, M. L.; Xue, W. A. Molecular Tracer Dynamics in Crystalline Organic Films at the Solid-Liquid Interface. J. Phys. Chem. B 2002, 106, 593-598.

(14) Abraham, M. J.; Murtola, T.; Schulz, R.; Pall, S.; Smith, J. C.; Hess, B.; Lindah, E. Gromacs: High Performance Molecular Simulations through Multi-Level Parallelism from Laptops to Supercomputers. SoftwareX 2015, 1-2, 19-25.

(15) Marrink, S. J.; Risselada, H. J.; Yefimov, S.; Tieleman, D. P.; de Vries, A. H. The MARTINI Force Field: Coarse Grained Model for Biomolecular Simulations. J. Phys. Chem. B 2007, 111, 7812-7824.

(16) Marrink, S. J.; Tieleman, D. P. Perspective on the Martini Model. Chem. Soc. Rev. 2013, 42, 6801-6822.

(17) Lim, R.; Li, J.; Li, S. F. Y.; Feng, Z.; Valiyaveettil, S. Formation of Two-Dimensional Supramolecular Chiral Lamellae by Diamide Molecules at the Solution/graphite Interface: A Scanning Tunneling Microscopy Study. Langmuir 2000, 16, 7023-7030.

(18) Marrink, S.-J.; Periole, X. Biomolecular Simulations. In Methods in Molecular Biology; Monticelli, L., Salonen, E., Eds.; Humana Press: Totowa, NJ, 2013; Vol. 924.

(19) Kolesnik, A. D.; Ratanov, N. Telegraph Processes and Option Pricing; Springer, 2013. 